\title{
Relativistic mechanics in a general setting
}

\author{
G. Sardanashvili \\ Department of Theoretical Physics, Moscow State University, Moscow, Russia
}

\begin{abstract}
Relativistic mechanics on an arbitrary manifold is formulated in the terms of jets of its one-dimensional submanifolds. A generic relativistic Lagrangian is constructed. Relativistic mechanics on a pseudo-Riemannian manifold is particularly considered.
\end{abstract}

\section{Introduction}

Classical non-relativistic mechanics is adequately formulated as Lagrangian and Hamiltonian theory on a fibre bundle $Q \rightarrow \mathbb{R}$ over the time axis $\mathbb{R}[1,3,6,8,9,11]$.

If a configuration space $Q$ of a mechanical system has no preferable fibration $Q \rightarrow \mathbb{R}$, we obtain a general formulation of relativistic mechanics, including Special Relativity on the Minkowski space $Q=\mathbb{R}^{4}[3,9,11]$. A velocity space of relativistic mechanics is the first order jet manifold $J_{1}^{1} Q$ of one-dimensional submanifolds of the configuration space $Q$. The notion of jets of submanifolds $[2,4,5,7]$ generalizes that of jets of sections of fibre bundles which are utilized in field theory and non-relativistic mechanics (Section 2). The jet bundle $J_{1}^{1} Q \rightarrow Q$ is projective, and one can think of its fibres as being spaces of the three-velocities of a relativistic system (Section 3). The four-velocities of a relativistic system are represented by elements of the tangent bundle $T Q$ of the configuration space $Q$, while the cotangent bundle $T^{*} Q$, endowed with the canonical symplectic form, plays a role of the phase space of relativistic theory (Section 6).

We develop Lagrangian formalism on the jet bundle $J_{1}^{1} Q \rightarrow Q$ (Section 4 ). We show that, in the framework of this formalism, Lagrangians possess a certain gauge symmetry (27) and, consequently, the corresponding Lagrange operators obey the rather restrictive Noether identity (28). Solving this Noether identity, we obtain the generic Lagrangian (31) and the equation of motion (51) of relativistic mechanics on a manifold $Q$. In particular, if $Q$ is the Minkowski space, we are in the case of Special Relativity (Example 4).

Generalizing this example, we consider relativistic mechanics on an arbitrary pseudoRiemannian manifold. Its equation of motion is the relativistic geodesic equation (55). Hamiltonian relativistic mechanics on a pseudo-Riemannian manifold is developed in Section 6. Its generic Hamiltonian takes the form (69). 


\section{Jets of submanifolds}

Jets of sections of fibre bundles are particular jets of submanifolds of a manifold $[2,4,5,7]$.

Given an $m$-dimensional smooth real manifold $Z$, a $k$-order jet of $n$-dimensional submanifolds of $Z$ at a point $z \in Z$ is defined as an equivalence class $j_{z}^{k} S$ of $n$-dimensional imbedded submanifolds of $Z$ through $z$ which are tangent to each other at $z$ with order $k \geq 0$. Namely, two submanifolds

$$
i_{S}: S \rightarrow Z, \quad i_{S^{\prime}}: S^{\prime} \rightarrow Z
$$

through a point $z \in Z$ belong to the same equivalence class $j_{z}^{k} S$ if and only if the images of the $k$-tangent morphisms

$$
T^{k} i_{S}: T^{k} S \rightarrow T^{k} Z, \quad T^{k} i_{S^{\prime}}: T^{k} S^{\prime} \rightarrow T^{k} Z
$$

coincide with each other. The set

$$
J_{n}^{k} Z=\bigcup_{z \in Z} j_{z}^{k} S
$$

of $k$-order jets of submanifolds is a finite-dimensional real smooth manifold, called the $k$-order jet manifold of submanifolds. For the sake of convenience, we put $J_{n}^{0} Z=Z$.

If $k>0$, let $Y \rightarrow X$ be an $m$-dimensional fibre bundle over an $n$-dimensional base $X$ and $J^{k} Y$ the $k$-order jet manifold of sections of $Y \rightarrow X$. Given an imbedding $\Phi: Y \rightarrow Z$, there is the natural injection

$$
J^{k} \Phi: J^{k} Y \rightarrow J_{n}^{k} Z, \quad j_{x}^{k} s \rightarrow[\Phi \circ s]_{\Phi(s(x))}^{k}
$$

where $s$ are sections of $Y \rightarrow X$. This injection defines a chart on $J_{n}^{k} Z$. These charts provide a manifold atlas of $J_{n}^{k} Z$.

Let us restrict our consideration to first order jets of submanifolds. There is obvious one-to-one correspondence

$$
\lambda_{(1)}: j_{z}^{1} S \rightarrow V_{j_{z}^{1} S} \subset T_{z} Z
$$

between the jets $j_{z}^{1} S$ at a point $z \in Z$ and the $n$-dimensional vector subspaces of the tangent space $T_{z} Z$ of $Z$ at $z$. It follows that $J_{n}^{1} Z$ is a fibre bundle

$$
\rho: J_{n}^{1} Z \rightarrow Z
$$

with the structure group $G L(n, m-n ; \mathbb{R})$ of linear transformations of the vector space $\mathbb{R}^{m}$ which preserve its subspace $\mathbb{R}^{n}$. The typical fibre of the fibre bundle (3) is the Grassmann manifold

$$
\mathfrak{G}(n, m-n ; \mathbb{R})=G L(m ; \mathbb{R}) / G L(n, m-n ; \mathbb{R}) .
$$


This fibre bundle possesses the following coordinate atlas.

Let $\left\{\left(U ; z^{A}\right)\right\}$ be a coordinate atlas of $Z$. Though $J_{n}^{0} Z=Z$, let us provide $J_{n}^{0} Z$ with an atlas where every chart $\left(U ; z^{A}\right)$ on a domain $U \subset Z$ is replaced with the

$$
\left(\begin{array}{c}
m \\
n
\end{array}\right)=\frac{m !}{n !(m-n) !}
$$

charts on the same domain $U$ which correspond to different partitions of the collection $\left(z^{1} \cdots z^{A}\right)$ in the collections of $n$ and $m-n$ coordinates

$$
\left(U ; x^{\lambda}, y^{i}\right), \quad \lambda=1, \ldots, n, \quad i=1, \ldots, m-n .
$$

The transition functions between the coordinate charts (4) of $J_{n}^{0} Z$ associated with a coordinate chart $\left(U, z^{A}\right)$ of $Z$ are reduced to exchange between coordinates $x^{\lambda}$ and $y^{i}$. Transition functions between arbitrary coordinate charts of the manifold $J_{n}^{0} Z$ take the form

$$
x^{\prime \lambda}=x^{\prime \lambda}\left(x^{\mu}, y^{k}\right), \quad y^{\prime i}=y^{\prime i}\left(x^{\mu}, y^{k}\right) .
$$

Given the coordinate atlas (4) - (5) of a manifold $J_{n}^{0} Z$, the first order jet manifold $J_{n}^{1} Z$ is endowed with an atlas of adapted coordinates

$$
\left(\rho^{-1}(U)=U \times \mathbb{R}^{(m-n) n} ; x^{\lambda}, y^{i}, y_{\lambda}^{i}\right),
$$

possessing transition functions

$$
y_{\lambda}^{\prime i}=\left(\frac{\partial y^{\prime i}}{\partial y^{j}} y_{\alpha}^{j}+\frac{\partial y^{\prime i}}{\partial x^{\alpha}}\right)\left(\frac{\partial x^{\alpha}}{\partial y^{\prime k}} y_{\lambda}^{\prime k}+\frac{\partial x^{\alpha}}{\partial x^{\prime \lambda}}\right) .
$$

\section{Relativistic mechanics}

As was mentioned above, a velocity space of relativistic mechanics is the first order jet manifold $J_{1}^{1} Q$ of one-dimensional submanifolds of a configuration space $Q[3,9,11]$.

Given an $m$-dimensional manifold $Q$ coordinated by $\left(q^{\lambda}\right)$, let us consider the jet manifold $J_{1}^{1} Q$ of its one-dimensional submanifolds. Let us provide $Q=J_{1}^{0} Q$ with the coordinates (4):

$$
\left(U ; x^{0}=q^{0}, y^{i}=q^{i}\right)=\left(U ; q^{\lambda}\right) .
$$

Then the jet manifold

$$
\rho: J_{1}^{1} Q \rightarrow Q
$$

is endowed with coordinates (6):

$$
\left(\rho^{-1}(U) ; q^{0}, q^{i}, q_{0}^{i}\right)
$$


possessing transition functions (5), (7) which read

$$
\begin{aligned}
& q^{\prime 0}=q^{\prime 0}\left(q^{0}, q^{k}\right), \quad q^{\prime 0}=q^{\prime 0}\left(q^{0}, q^{k}\right), \\
& q_{0}^{\prime i}=\left(\frac{\partial q^{\prime i}}{\partial q^{j}} q_{0}^{j}+\frac{\partial q^{i}}{\partial q^{0}}\right)\left(\frac{\partial q^{0}}{\partial q^{j}} q_{0}^{j}+\frac{\partial q^{0}}{\partial q^{0}}\right)^{-1} .
\end{aligned}
$$

A glance at the transformation law (11) shows that $J_{1}^{1} Q \rightarrow Q$ is a fibre bundle in projective spaces.

Example 1. Let $Q=M^{4}=\mathbb{R}^{4}$ be a Minkowski space whose Cartesian coordinates $\left(q^{\lambda}\right), \lambda=0,1,2,3$, are subject to the Lorentz transformations (10):

$$
q^{\prime 0}=q^{0} \operatorname{ch} \alpha-q^{1} \operatorname{sh} \alpha, \quad q^{\prime 1}=-q^{0} \operatorname{sh} \alpha+q^{1} \operatorname{ch} \alpha, \quad q^{\prime 2,3}=q^{2,3} .
$$

Then $q^{i}(11)$ are exactly the Lorentz transformations

$$
q_{0}^{\prime 1}=\frac{q_{0}^{1} \operatorname{ch} \alpha-\operatorname{sh} \alpha}{-q_{0}^{1} \operatorname{sh} \alpha+\operatorname{ch} \alpha} \quad q_{0}^{\prime 2,3}=\frac{q_{0}^{2,3}}{-q_{0}^{1} \operatorname{sh} \alpha+\operatorname{ch} \alpha}
$$

of three-velocities in relativistic mechanics $[9,11]$.

In view of Example 1, one can think of the velocity space $J_{1}^{1} Q$ of relativistic mechanics as being a space of three-velocities. For the sake of convenience, we agree to call $J_{1}^{1} Q$ the three-velocity space and its coordinate transformations $(10)-(11)$ the relativistic transformations, though a dimension of $Q$ need not equal $3+1$.

\section{Lagrangian relativistic mechanics}

Given the coordinate chart (9) of $J_{1}^{1} Q$, one can regard $\rho^{-1}(U) \subset J_{1}^{1} Q$ as the first order jet manifold $J^{1} U$ of sections of the fibre bundle

$$
\pi: U \ni\left(q^{0}, q^{i}\right) \rightarrow\left(q^{0}\right) \in \pi(U) \subset \mathbb{R} .
$$

Then three-velocities $\left(q_{0}^{i}\right) \in \rho^{-1}(U)$ of a relativistic system on $U$ can be treated as absolute velocities of a local non-relativistic system on the configuration space $U$ (13). However, this treatment is broken under the relativistic transformations $q_{0}^{i} \rightarrow q_{0}^{i}(10)$ since they are not affine. One can develop first order Lagrangian formalism with a Lagrangian

$$
L=\mathcal{L} d q^{0} \in \mathcal{O}^{0,1}\left(\rho^{-1}(U)\right)
$$

on a coordinate chart $\rho^{-1}(U)$, but this Lagrangian fails to be globally defined on $J_{1}^{1} Q$ (see Remark 3 below). The graded differential algebra $\mathcal{O}^{*}\left(\rho^{-1}(U)\right)$ of exterior forms on $\rho^{-1}(U)$ is generated by horizontal forms $d q^{0}$ and contact forms $d q^{i}-q_{0}^{i} d q^{0}$. Coordinate transformations (10) preserve the ideal of contact forms, but horizontal forms are not transformed

into horizontal forms, unless coordinate transition functions $q^{0}(10)$ are independent of coordinates $q^{i}$. 
In order to overcome this difficulty, let us consider a trivial fibre bundle

$$
Q_{R}=\mathbb{R} \times Q \rightarrow \mathbb{R},
$$

whose base $\mathbb{R}$ is endowed with a Cartesian coordinate $\tau$ [5]. This fibre bundle is provided with an atlas of coordinate charts

$$
\left(\mathbb{R} \times U ; \tau, q^{\lambda}\right),
$$

where $\left(U ; q^{0}, q^{i}\right)$ are the coordinate charts (8) of the manifold $J_{1}^{0} Q$. The coordinate charts (15) possess transition functions (10). Let $J^{1} Q_{R}$ be the first order jet manifold of the fibre bundle (14). Since the trivialization (14) is fixed, there is the canonical isomorphism of $J^{1} Q_{R}$ to the vertical tangent bundle

$$
J^{1} Q_{R}=V Q_{R}=\mathbb{R} \times T Q
$$

of $Q_{R} \rightarrow \mathbb{R}[5,6]$.

Given the coordinate atlas (15) of $Q_{R}$, the jet manifold $J^{1} Q_{R}$ is endowed with the coordinate charts

$$
\left(\left(\pi^{1}\right)^{-1}(\mathbb{R} \times U)=\mathbb{R} \times U \times \mathbb{R}^{m} ; \tau, q^{\lambda}, q_{\tau}^{\lambda}\right),
$$

possessing transition functions

$$
q_{\tau}^{\prime \lambda}=\frac{\partial q^{\prime \lambda}}{\partial q^{\mu}} q_{\tau}^{\mu} .
$$

Relative to the coordinates (17), the isomorphism (16) takes the form

$$
\left(\tau, q^{\mu}, q_{\tau}^{\mu}\right) \rightarrow\left(\tau, q^{\mu}, \dot{q}^{\mu}=q_{\tau}^{\mu}\right)
$$

Example 2. Let $Q=M^{4}$ be a Minkowski space in Example 1 whose Cartesian coordinates $\left(q^{0}, q^{i}\right)$ are subject to the Lorentz transformations (12). Then the corresponding transformations (18) take the form

$$
q_{\tau}^{\prime 0}=q_{\tau}^{0} \operatorname{ch} \alpha-q_{\tau}^{1} \operatorname{sh} \alpha, \quad q_{\tau}^{\prime 1}=-q_{\tau}^{0} \operatorname{sh} \alpha+q_{\tau}^{1} \operatorname{ch} \alpha, \quad q_{\tau}^{\prime 2,3}=q_{\tau}^{2,3}
$$

of transformations of four-velocities in relativistic mechanics.

In view of Example 2, we agree to call fibre elements of $J^{1} Q_{R} \rightarrow Q_{R}$ the four-velocities though the dimension of $Q$ need not equal 4. Due to the canonical isomorphism $q_{\tau}^{\lambda} \rightarrow \dot{q}^{\lambda}$ (16), by four-velocities also are meant the elements of the tangent bundle $T Q$, which is called the space of four-velocities.

Obviously, the non-zero jet (19) of sections of the fibre bundle (14) defines some jet of one-dimensional subbundles of the manifold $\{\tau\} \times Q$ through a point $\left(q^{0}, q^{i}\right) \in Q$, but this is not one-to-one correspondence. 
Since non-zero elements of $J^{1} Q_{R}$ characterize jets of one-dimensional submanifolds of $Q$, one hopes to describe the dynamics of one-dimensional submanifolds of a manifold $Q$ as that of sections of the fibre bundle (14). For this purpose, let us refine the relation between elements of the jet manifolds $J_{1}^{1} Q$ and $J^{1} Q_{R}$.

Let us consider the manifold product $\mathbb{R} \times J_{1}^{1} Q$. It is a fibre bundle over $Q_{R}$. Given a coordinate atlas (15) of $Q_{R}$, this product is endowed with the coordinate charts

$$
\left(U_{R} \times \rho^{-1}(U)=U_{R} \times U \times \mathbb{R}^{m-1} ; \tau, q^{0}, q^{i}, q_{0}^{i}\right),
$$

possessing transition functions (10) - (11). Let us assign to an element $\left(\tau, q^{0}, q^{i}, q_{0}^{i}\right)$ of the chart (20) the elements $\left(\tau, q^{0}, q^{i}, q_{\tau}^{0}, q_{\tau}^{i}\right)$ of the chart (17) whose coordinates obey the relations

$$
q_{0}^{i} q_{\tau}^{0}=q_{\tau}^{i} .
$$

These elements make up a one-dimensional vector space. The relations (21) are maintained under coordinate transformations (11) and (18) [4,5]. Thus, one can associate:

$$
\left(\tau, q^{0}, q^{i}, q_{0}^{i}\right) \rightarrow\left\{\left(\tau, q^{0}, q^{i}, q_{\tau}^{0}, q_{\tau}^{i}\right) \mid q_{0}^{i} q_{\tau}^{0}=q_{\tau}^{i}\right\},
$$

to each element of the manifold $\mathbb{R} \times J_{1}^{1} Q$ a one-dimensional vector space in the jet manifold $J^{1} Q_{R}$. This is a subspace of elements

$$
q_{\tau}^{0}\left(\partial_{0}+q_{0}^{i} \partial_{i}\right)
$$

of a fibre of the vertical tangent bundle (16) at a point $\left(\tau, q^{0}, q^{i}\right)$. Conversely, given a non-zero element (19) of $J^{1} Q_{R}$, there is a coordinate chart (17) such that this element defines a unique element of $\mathbb{R} \times J_{1}^{1} Q$ by the relations

$$
q_{0}^{i}=\frac{q_{\tau}^{i}}{q_{\tau}^{0}} .
$$

Thus, we have shown the following. Let $\left(\tau, q^{\lambda}\right)$ further be arbitrary coordinates on the product $Q_{R}(14)$ and $\left(\tau, q^{\lambda}, q_{\tau}^{\lambda}\right)$ the corresponding coordinates on the jet manifold $J^{1} Q_{R}$.

Theorem 1. (i) Any jet of submanifolds through a point $q \in Q$ defines some (but not unique) jet of sections of the fibre bundle $Q_{R}$ (14) through a point $\tau \times q$ for any $\tau \in \mathbb{R}$ in accordance with the relations (21).

(ii) Any non-zero element of $J^{1} Q_{R}$ defines a unique element of the jet manifold $J_{1}^{1} Q$ by means of the relations (23). However, non-zero elements of $J^{1} Q_{R}$ can correspond to different jets of submanifolds.

(iii) Two elements $\left(\tau, q^{\lambda}, q_{\tau}^{\lambda}\right)$ and $\left(\tau, q^{\lambda}, q_{\tau}^{\prime \lambda}\right)$ of $J^{1} Q_{R}$ correspond to the same jet of submanifolds if $q_{\tau}^{\prime \lambda}=r q_{\tau}^{\lambda}, r \in \mathbb{R} \backslash\{0\}$.

In the case of a Minkowski space $Q=M^{4}$ in Examples 1 and 2, the equalities (21) and (23) are the familiar relations between three- and four-velocities. 
Based on Theorem 1, we can develop Lagrangian theory of one-dimensional submanifolds of a manifold $Q$ as that of sections of the fibre bundle $Q_{R}(14)$. Let

$$
L=\mathcal{L}\left(\tau, q^{\lambda}, q_{\tau}^{\lambda}\right) d \tau,
$$

be a first order Lagrangian on the jet manifold $J^{1} Q_{R}$. The corresponding Lagrange operator reads

$$
\delta L=\mathcal{E}_{\lambda} d q^{\lambda} \wedge d \tau, \quad \mathcal{E}_{\lambda}=\partial_{\lambda} \mathcal{L}-d_{\tau} \partial_{\lambda}^{\tau} \mathcal{L} .
$$

It yields the Lagrange equation

$$
\mathcal{E}_{\lambda}=\partial_{\lambda} \mathcal{L}-d_{\tau} \partial_{\lambda}^{\tau} \mathcal{L}=0 .
$$

In accordance with Theorem 1, it seems reasonable to require that, in order to describe jets of one-dimensional submanifolds of $Q$, the Lagrangian $L(24)$ on $J^{1} Q_{R}$ possesses a gauge symmetry given by vector fields $u=\chi(\tau) \partial_{\tau}$ on $Q_{R}$ or, equivalently, their vertical part

$$
u_{V}=-\chi q_{\tau}^{\lambda} \partial_{\lambda},
$$

which are generalized vector fields on $Q_{R}[5,6]$. Then the variational derivatives of this Lagrangian obey the Noether identity:

$$
q_{\tau}^{\lambda} \mathcal{E}_{\lambda}=0 .
$$

We call such a Lagrangian the relativistic Lagrangian.

In order to obtain a generic form of a relativistic Lagrangian $L$, let us regard the Noether identity (28) as an equation for $L$. It admits the following solution. Let

$$
\frac{1}{2 N !} G_{\alpha_{1} \ldots \alpha_{2 N}}\left(q^{\nu}\right) d q^{\alpha_{1}} \vee \cdots \vee d q^{\alpha_{2 N}}
$$

be a symmetric tensor field on $Q$ such that the function

$$
G=G_{\alpha_{1} \ldots \alpha_{2 N}}\left(q^{\nu}\right) \dot{q}^{\alpha_{1}} \cdots \dot{q}^{\alpha_{2 N}}
$$

is positive:

$$
G>0,
$$

everywhere on $T Q \backslash \widehat{0}(Q)$. Let $A=A_{\mu}\left(q^{\nu}\right) d q^{\mu}$ be a one-form on $Q$. Given the pull-back of $G$ and $A$ onto $J^{1} Q_{R}$ due to the canonical isomorphism (16), we define a Lagrangian

$$
L=\left(G^{1 / 2 N}+q_{\tau}^{\mu} A_{\mu}\right) d \tau, \quad G=G_{\alpha_{1} \ldots \alpha_{2 N}} q_{\tau}^{\alpha_{1}} \cdots q_{\tau}^{\alpha_{2 N}},
$$


on $J^{1} Q_{R} \backslash(\mathbb{R} \times \widehat{0}(Q))$ where $\hat{0}$ is the global zero section of $T Q \rightarrow Q$. The corresponding Lagrange equation reads

$$
\begin{aligned}
\mathcal{E}_{\lambda}= & \frac{\partial_{\lambda} G}{2 N G^{1-1 / 2 N}}-d_{\tau}\left(\frac{\partial_{\lambda}^{\tau} G}{2 N G^{1-1 / 2 N}}\right)+F_{\lambda \mu} q_{\tau}^{\mu}= \\
& E_{\beta}\left[\delta_{\lambda}^{\beta}-q_{\tau}^{\beta} G_{\lambda \nu_{2} \ldots \nu_{2 N}} q_{\tau}^{\nu_{2}} \cdots q_{\tau}^{\nu_{2 N}} G^{-1}\right] G^{1 / 2 N-1}=0, \\
E_{\beta}= & \left(\frac{\partial_{\beta} G_{\mu \alpha_{2} \ldots \alpha_{2 N}}}{2 N}-\partial_{\mu} G_{\beta \alpha_{2} \ldots \alpha_{2 N}}\right) q_{\tau}^{\mu} q_{\tau}^{\alpha_{2}} \cdots q_{\tau}^{\alpha_{2 N}}- \\
& (2 N-1) G_{\beta \mu \alpha_{3} \ldots \alpha_{2 N}} q_{\tau \tau}^{\mu} q_{\tau}^{\alpha_{3}} \cdots q_{\tau}^{\alpha_{2 N}}+G^{1-1 / 2 N} F_{\beta \mu} q_{\tau}^{\mu}, \\
F_{\lambda \mu}= & \partial_{\lambda} A_{\mu}-\partial_{\mu} A_{\lambda} .
\end{aligned}
$$

It is readily observed that the variational derivatives $\mathcal{E}_{\lambda}(32)$ satisfy the Noether identity (28). Moreover, any relativistic Lagrangian obeying the Noether identity (28) is of type (31).

A glance at the Lagrange equation (32) shows that it holds if

$$
E_{\beta}=\Phi G_{\beta \nu_{2} \ldots \nu_{2 N}} q_{\tau}^{\nu_{2}} \cdots q_{\tau}^{\nu_{2 N}} G^{-1}
$$

where $\Phi$ is some function on $J^{1} Q_{R}$. In particular, we consider the equation

$$
E_{\beta}=0 .
$$

Because of the Noether identity (28), the system of equations (32) is underdetermined. To overcome this difficulty, one can complete it with some additional equation. Given the function $G$ (31), let us choose the condition

$$
G=1 \text {. }
$$

Owing to the property (30), the function $G(31)$ possesses a nowhere vanishing differential. Therefore, its level surface $W_{G}$ defined by the condition (36) is a submanifold of $J^{1} Q_{R}$.

Our choice of the equation (35) and the condition (36) is motivated by the following facts.

LEmma 2. Any solution of the Lagrange equation (32) living in the submanifold $W_{G}$ is a solution of the equation (35).

Proof. A solution of the Lagrange equation (32) living in the submanifold $W_{G}$ obeys the system of equations

$$
\mathcal{E}_{\lambda}=0, \quad G=1
$$

Therefore, it satisfies the equality

$$
d_{\tau} G=0
$$


Then a glance at the expression (32) shows that the equations (37) are equivalent to the equations

$$
\begin{aligned}
E_{\lambda}= & \left(\frac{\partial_{\lambda} G_{\mu \alpha_{2} \ldots \alpha_{2 N}}}{2 N}-\partial_{\mu} G_{\lambda \alpha_{2} \ldots \alpha_{2 N}}\right) q_{\tau}^{\mu} q_{\tau}^{\alpha_{2}} \cdots q_{\tau}^{\alpha_{2 N}}- \\
& (2 N-1) G_{\beta \mu \alpha_{3} \ldots \alpha_{2 N}} q_{\tau \tau}^{\mu} q_{\tau}^{\alpha_{3}} \cdots q_{\tau}^{\alpha_{2 N}}+F_{\beta \mu} q_{\tau}^{\mu}=0 \\
G= & G_{\alpha_{1} \ldots \alpha_{2 N}} q_{\tau}^{\alpha_{1}} \cdots q_{\tau}^{\alpha_{2 N}}=1 .
\end{aligned}
$$

LEMma 3. Solutions of the equation (35) do not leave the submanifold $W_{G}(36)$.

\section{Proof. Since}

$$
d_{\tau} G=-\frac{2 N}{2 N-1} q_{\tau}^{\beta} E_{\beta},
$$

any solution of the equation (35) intersecting the submanifold $W_{G}(36)$ obeys the equality (38) and, consequently, lives in $W_{G}$.

QED

The system of equations (39) is called the relativistic equation. Its components $E_{\lambda}$ (33) are not independent, but obeys the relation

$$
q_{\tau}^{\beta} E_{\beta}=-\frac{2 N-1}{2 N} d_{\tau} G=0, \quad G=1,
$$

similar to the Noether identity (28). The condition (36) is called the relativistic constraint.

Though the equation (32) for sections of a fibre bundle $Q_{R} \rightarrow \mathbb{R}$ is underdetermined, it is determined if, given a coordinate chart $\left(U ; q^{0}, q^{i}\right)(8)$ of $Q$ and the corresponding coordinate chart (15) of $Q_{R}$, we rewrite it in the terms of three-velocities $q_{0}^{i}(23)$ as an equation for sections of a fibre bundle $U \rightarrow \pi(U)(13)$.

Let us denote

$$
\bar{G}\left(q^{\lambda}, q_{0}^{i}\right)=\left(q_{\tau}^{0}\right)^{-2 N} G\left(q^{\lambda}, q_{\tau}^{\lambda}\right), \quad q_{\tau}^{0} \neq 0 .
$$

Then we have

$$
\mathcal{E}_{i}=q_{\tau}^{0}\left[\frac{\partial_{i} \bar{G}}{2 N \bar{G}^{1-1 / 2 N}}-\left(q_{\tau}^{0}\right)^{-1} d_{\tau}\left(\frac{\partial_{i}^{0} \bar{G}}{2 N \bar{G}^{1-1 / 2 N}}\right)+F_{i j} q_{0}^{j}+F_{i 0}\right] .
$$

Let us consider a solution $\left\{s^{\lambda}(\tau)\right\}$ of the equation (32) such that $\partial_{\tau} s^{0}$ does not vanish and there exists an inverse function $\tau\left(q^{0}\right)$. Then this solution can be represented by sections

$$
s^{i}(\tau)=\left(\bar{s}^{i} \circ s^{0}\right)(\tau)
$$

of the composite bundle

$$
\mathbb{R} \times U \rightarrow \mathbb{R} \times \pi(U) \rightarrow \mathbb{R}
$$


where $\bar{s}^{i}\left(q^{0}\right)=s^{i}\left(\tau\left(q^{0}\right)\right)$ are sections of $U \rightarrow \pi(U)$ and $s^{0}(\tau)$ are sections of $\mathbb{R} \times \pi(U) \rightarrow \mathbb{R}$. Restricted to such solutions, the equation (32) is equivalent to the equation

$$
\begin{aligned}
& \overline{\mathcal{E}}_{i}=\frac{\partial_{i} \bar{G}}{2 N \bar{G}^{1-1 / 2 N}}-d_{0}\left(\frac{\partial_{i}^{0} \bar{G}}{2 N \bar{G}^{1-1 / 2 N}}\right)+ \\
& \quad F_{i j} q_{0}^{j}+F_{i 0}=0, \\
& \overline{\mathcal{E}}_{0}=-q_{0}^{i} \overline{\mathcal{E}}_{i} .
\end{aligned}
$$

for sections $\bar{s}^{i}\left(q^{0}\right)$ of a fibre bundle $U \rightarrow \pi(U)$.

It is readily observed that the equation (42) is the Lagrange equation of the Lagrangian

$$
\bar{L}=\left(\bar{G}^{1 / 2 N}+q_{0}^{i} A_{i}+A_{0}\right) d q^{0}
$$

on the jet manifold $J^{1} U$ of a fibre bundle $U \rightarrow \pi(U)$.

Remark 3. Both the equation (42) and the Lagrangian (43) are defined only on a coordinate chart (8) of $Q$ since they are not maintained by transition functions (10)(11).

A solution $\bar{s}^{i}\left(q^{0}\right)$ of the equation (42) defines a solution $s^{\lambda}(\tau)$ (41) of the equation (32) up to an arbitrary function $s^{0}(\tau)$. The relativistic constraint (36) enables one to overcome this ambiguity as follows.

Let us assume that, restricted to the coordinate chart $\left(U ; q^{0}, q^{i}\right)$ (8) of $Q$, the relativistic constraint (36) has no solution $q_{\tau}^{0}=0$. Then it is brought into the form

$$
\left(q_{\tau}^{0}\right)^{2 N} \bar{G}\left(q^{\lambda}, q_{0}^{i}\right)=1
$$

where $\bar{G}$ is the function (40). With the condition (44), every three-velocity $\left(q_{0}^{i}\right)$ defines a unique pair of four-velocities

$$
q_{\tau}^{0}= \pm\left(\bar{G}\left(q^{\lambda}, q_{0}^{i}\right)\right)^{1 / 2 N}, \quad q_{\tau}^{i}=q_{\tau}^{0} q_{0}^{i} .
$$

Accordingly, any solution $\bar{s}^{i}\left(q^{0}\right)$ of the equation (42) leads to solutions

$$
\tau\left(q^{0}\right)= \pm \int\left(\bar{G}\left(q^{0}, \bar{s}^{i}\left(q^{0}\right), \partial_{0} \bar{s}^{i}\left(q_{0}\right)\right)^{-1 / 2 N} d q^{0}, \quad s^{i}(\tau)=s^{0}(\tau)\left(\partial_{i} \bar{s}^{i}\right)\left(s^{0}(\tau)\right)\right.
$$

of the equation (37) and, equivalently, the relativistic equation (39).

Example 4. Let $Q=M^{4}$ be a Minkowski space provided with the Minkowski metric $\eta_{\mu \nu}$ of signature $(+,---)$. This is the case of Special Relativity. Let $\mathcal{A}_{\lambda} d q^{\lambda}$ be a one-form on $Q$. Then

$$
L=\left[m\left(\eta_{\mu \nu} q_{\tau}^{\mu} q_{\tau}^{\nu}\right)^{1 / 2}+e \mathcal{A}_{\mu} q_{\tau}^{\mu}\right] d \tau, \quad m, e \in \mathbb{R},
$$

is a relativistic Lagrangian on $J^{1} Q_{R}$ which satisfies the Noether identity (28). The corresponding relativistic equation (39) reads

$$
\begin{aligned}
& m \eta_{\mu \nu} q_{\tau \tau}^{\nu}-e F_{\mu \nu} q_{\tau}^{\nu}=0, \\
& \eta_{\mu \nu} q_{\tau}^{\mu} q_{\tau}^{\nu}=1 .
\end{aligned}
$$


This describes a relativistic massive charge in the presence of an electromagnetic field $\mathcal{A}$. It follows from the relativistic constraint $(48)$ that $\left(q_{\tau}^{0}\right)^{2} \geq 1$. Therefore, passing to three-velocities, we obtain the Lagrangian (43):

$$
\bar{L}=\left[m\left(1-\sum_{i}\left(q_{0}^{i}\right)^{2}\right)^{1 / 2}+e\left(\mathcal{A}_{i} q_{0}^{i}+\mathcal{A}_{0}\right)\right] d q^{0},
$$

and the Lagrange equation (42):

$$
d_{0}\left(\frac{m q_{0}^{i}}{\left(1-\sum_{i}\left(q_{0}^{i}\right)^{2}\right)^{1 / 2}}\right)+e\left(F_{i j} q_{0}^{j}+F_{i 0}\right)=0 .
$$

Example 5. Let $Q=\mathbb{R}^{4}$ be an Euclidean space provided with the Euclidean metric $\epsilon$. This is the case of Euclidean Special Relativity. Let $\mathcal{A}_{\lambda} d q^{\lambda}$ be a one-form on $Q$. Then

$$
L=\left[\left(\epsilon_{\mu \nu} q_{\tau}^{\mu} q_{\tau}^{\nu}\right)^{1 / 2}+\mathcal{A}_{\mu} q_{\tau}^{\mu}\right] d \tau
$$

is a relativistic Lagrangian on $J^{1} Q_{R}$ which satisfies the Noether identity (28). The corresponding relativistic equation (39) reads

$$
\begin{aligned}
& m \epsilon_{\mu \nu} q_{\tau \tau}^{\nu}-e F_{\mu \nu} q_{\tau}^{\nu}=0, \\
& \epsilon_{\mu \nu} q_{\tau}^{\mu} q_{\tau}^{\nu}=1 .
\end{aligned}
$$

It follows from the relativistic constraint (50) that $0 \leq\left(q_{\tau}^{0}\right)^{2} \leq 1$. Passing to threevelocities, one therefore meets a problem.

\section{Relativistic geodesic equations}

A glance at the relativistic Lagrangian (31) shows that, because of the gauge symmetry (27), this Lagrangian is independent of $\tau$ and, therefore, it describes an autonomous mechanical system. Accordingly, the relativistic equation (39) on $Q_{R}$ is conservative and, therefore, it is equivalent to an autonomous second order equation on $Q$ whose solutions are parameterized by the coordinate $\tau$ on a base $\mathbb{R}$ of $Q_{R}$. Given holonomic coordinates $\left(q^{\lambda}, \dot{q}^{\lambda}, \ddot{q}^{\lambda}\right)$ of the second tangent bundle $T^{2} Q$, this autonomous second order equation (called the autonomous relativistic equation) reads

$$
\begin{aligned}
& \left(\frac{\partial_{\lambda} G_{\mu \alpha_{2} \ldots \alpha_{2 N}}}{2 N}-\partial_{\mu} G_{\lambda \alpha_{2} \ldots \alpha_{2 N}}\right) \dot{q}^{\mu} \dot{q}^{\alpha_{2}} \cdots \dot{q}^{\alpha_{2 N}}- \\
& \quad(2 N-1) G_{\beta \mu \alpha_{3} \ldots \alpha_{2 N}} \ddot{q}^{\mu} \dot{q}^{\alpha_{3}} \cdots \dot{q}^{\alpha_{2 N}}+F_{\beta \mu} \dot{q}^{\mu}=0 \\
& G=G_{\alpha_{1} \ldots \alpha_{2 N}} \dot{q}^{\alpha_{1}} \cdots \dot{q}^{\alpha_{2 N}}=1 .
\end{aligned}
$$

Due to the canonical isomorphism $q_{\tau}^{\lambda} \rightarrow \dot{q}^{\lambda}(16)$, the tangent bundle $T Q$ is regarded as a space of four-velocities. 
Generalizing Example 4, let us investigate relativistic mechanics on a four-dimensional pseudo-Riemannian manifold $Q=X$, coordinated by $\left(x^{\lambda}\right)$ and provided with a pseudoRiemannian metric $g$ of signature $(+,---)$. We agree to call $X$ a world manifold. Let $A=A_{\lambda} d x^{\lambda}$ be a one-form on $X$. Let us consider the relativistic Lagrangian (31):

$$
L=\left[\left(g_{\alpha \beta} x_{\tau}^{\alpha} x_{\tau}^{\beta}\right)^{1 / 2}+A_{\mu} x_{\tau}^{\mu}\right] d \tau
$$

and the relativistic constraint (36):

$$
g_{\alpha \beta} x_{\tau}^{\alpha} x_{\tau}^{\beta}=1
$$

The corresponding autonomous relativistic equation (39) on $X$ takes the form

$$
\begin{aligned}
& \ddot{x}^{\lambda}-\left\{\mu^{\lambda}{ }_{\nu}\right\} \dot{x}^{\mu} \dot{x}^{\nu}-g^{\lambda \beta} F_{\beta \nu} \dot{x}^{\nu}=0, \\
& g=g_{\alpha \beta} \dot{x}^{\alpha} \dot{x}^{\beta}=1
\end{aligned}
$$

where $\left\{\mu^{\lambda} \nu\right\}$ is the Levi-Civita connection. A glance at the equality (52) shows that it is a geodesic equation on $T X$ with respect to an affine connection

$$
K_{\mu}^{\lambda}=\left\{\mu^{\lambda}{ }_{\nu}\right\} \dot{x}^{\nu}+g^{\lambda \nu} F_{\nu \mu}
$$

on $T X$.

A particular form of this connection follows from the fact that the geodesic equation (52) is derived from a Lagrange equation, i.e., we are in the case of Lagrangian relativistic mechanics. In a general setting, relativistic mechanics on a pseudo-Riemannian manifold $(X, g)$ can be formulated as follows.

The geodesic equation

$$
\ddot{x}^{\mu}=K_{\lambda}^{\mu}\left(x^{\nu}, \dot{x}^{\nu}\right) \dot{x}^{\lambda},
$$

on the tangent bundle $T X$ with respect to a connection

$$
K=d x^{\lambda} \otimes\left(\partial_{\lambda}+K_{\lambda}^{\mu} \dot{\partial}_{\mu}\right)
$$

on $T X \rightarrow X$ is called a relativistic geodesic equation if a geodesic vector field of $K$ lives in the subbundle of hyperboloids

$$
W_{g}=\left\{\dot{x}^{\lambda} \in T X \mid g_{\lambda \mu} \dot{x}^{\lambda} \dot{x}^{\mu}=1\right\} \subset T X
$$

defined by the relativistic constraint (53).

One can show that the equation (55) is a relativistic geodesic equation if the condition

$$
\left(\partial_{\lambda} g_{\mu \nu} \dot{x}^{\mu}+2 g_{\mu \nu} K_{\lambda}^{\mu}\right) \dot{x}^{\lambda} \dot{x}^{\nu}=0
$$

holds. 
Obviously, the connection (54) fulfils the condition (58). Any metric connection, e.g., the Levi-Civita connection $\left\{\lambda^{\mu}{ }_{\nu}\right\}$ on $T X$ satisfies the condition (58).

Given a Levi-Civita connection $\left\{\lambda^{\mu}{ }_{\nu}\right\}$, any connection $K$ on $T X \rightarrow X$ can be written as

$$
K_{\lambda}^{\mu}=\left\{\lambda^{\mu}{ }_{\nu}\right\} \dot{x}^{\nu}+\sigma_{\lambda}^{\mu}\left(x^{\lambda}, \dot{x}^{\lambda}\right)
$$

where

$$
\sigma=\sigma_{\lambda}^{\mu} d x^{\lambda} \otimes \dot{\partial}_{\lambda}
$$

is some soldering form on $T X$. Then the condition (58) takes the form

$$
g_{\mu \nu} \sigma_{\lambda}^{\mu} \dot{x}^{\lambda} \dot{x}^{\nu}=0
$$

With the decomposition (59), one can think of the relativistic geodesic equation (55):

$$
\ddot{x}^{\mu}=\left\{{ }_{\lambda}{ }^{\mu}{ }_{\nu}\right\} \dot{x}^{\nu} \dot{x}^{\lambda}+\sigma_{\lambda}^{\mu}\left(x^{\lambda}, \dot{x}^{\lambda}\right) \dot{x}^{\lambda},
$$

as describing a relativistic particle in the presence of a gravitational field $g$ and a nongravitational external force $\sigma$.

\section{Hamiltonian relativistic mechanics}

We are in the case of relativistic mechanics on a pseudo-Riemmanian world manifold $(X, g)$. Given the coordinate chart (13) of its configuration space $X$, the homogeneous Legendre bundle corresponding to the local non-relativistic system on $U$ is the cotangent bundle $T^{*} U$ of $U$. This fact motivate us to think of the cotangent bundle $T^{*} X$ as being the phase space of relativistic mechanics on $X$. It is provided with the canonical symplectic form

$$
\Omega=d p_{\lambda} \wedge d x^{\lambda}
$$

and the corresponding Poisson bracket $\{$,$\} .$

A relativistic Hamiltonian is defined as follows $[9,10,11]$. Let $H$ be a smooth real function on $T^{*} X$ such that the morphism

$$
\widetilde{H}: T^{*} X \rightarrow T X, \quad \dot{x}^{\mu} \circ \widetilde{H}=\partial^{\mu} H,
$$

is a bundle isomorphism. Then the inverse image

$$
N=\widetilde{H}^{-1}\left(W_{g}\right)
$$

of the subbundle of hyperboloids $W_{g}(57)$ is a one-codimensional (consequently, coisotropic) closed imbedded subbundle $N$ of $T^{*} X$ given by the condition

$$
H_{T}=g_{\mu \nu} \partial^{\mu} H \partial^{\nu} H-1=0 .
$$


We say that $H$ is a relativistic Hamiltonian if the Poisson bracket $\left\{H, H_{T}\right\}$ vanishes on $N$. This means that the Hamiltonian vector field

$$
\gamma=\partial^{\lambda} H \partial_{\lambda}-\partial_{\lambda} H \partial^{\lambda}
$$

of $H$ preserves the constraint $N$ and, restricted to $N$, it obeys the equation

$$
\gamma\rfloor \Omega_{N}+i_{N}^{*} d H=0
$$

which is the Hamilton equation of a Dirac constrained system on $N$ with a Hamiltonian $H[3]$.

The morphism (64) sends the vector field $\gamma(66)$ onto the vector field

$$
\gamma_{T}=\dot{x}^{\lambda} \partial_{\lambda}+\left(\partial^{\mu} H \partial^{\lambda} \partial_{\mu} H-\partial_{\mu} H \partial^{\lambda} \partial^{\mu} H\right) \dot{\partial}_{\lambda}
$$

on $T X$. This vector field defines the autonomous second order dynamic equation

$$
\ddot{x}^{\lambda}=\partial^{\mu} H \partial^{\lambda} \partial_{\mu} H-\partial_{\mu} H \partial^{\lambda} \partial^{\mu} H
$$

on $X$ which preserves the subbundle of hyperboloids (57), i.e., it is the autonomous relativistic equation (51).

Example 6. The following is a basic example of relativistic Hamiltonian mechanics. Given a one-form $A=A_{\mu} d q^{\mu}$ on $X$, let us put

$$
H=g^{\mu \nu}\left(p_{\mu}-A_{\mu}\right)\left(p_{\nu}-A_{\nu}\right) .
$$

Then $H_{T}=2 H-1$ and, hence, $\left\{H, H_{T}\right\}=0$. The constraint $H_{T}=0$ (65) defines a one-codimensional closed imbedded subbundle $N$ of $T^{*} X$. The Hamilton equation (67) takes the form $\gamma\rfloor \Omega_{N}=0$. Its solution (66) reads

$$
\begin{aligned}
& \dot{x}^{\alpha}=g^{\alpha \nu}\left(p_{\nu}-A_{\nu}\right), \\
& \dot{p}_{\alpha}=-\frac{1}{2} \partial_{\alpha} g^{\mu \nu}\left(p_{\mu}-A_{\mu}\right)\left(p_{\nu}-A_{\nu}\right)+g^{\mu \nu}\left(p_{\mu}-A_{\mu}\right) \partial_{\alpha} A_{\nu} .
\end{aligned}
$$

The corresponding autonomous second order dynamic equation (68) on $X$ is

$$
\begin{aligned}
& \ddot{x}^{\lambda}-\left\{\mu^{\lambda}{ }_{\nu}\right\} \dot{x}^{\mu} \dot{x}^{\nu}-g^{\lambda \nu} F_{\nu \mu} \dot{x}^{\mu}=0 \\
& \left\{\mu^{\lambda} \nu\right\}=-\frac{1}{2} g^{\lambda \beta}\left(\partial_{\mu} g_{\beta \nu}+\partial_{\nu} g_{\beta \mu}-\partial_{\beta} g_{\mu \nu}\right) \\
& F_{\mu \nu}=\partial_{\mu} A_{\nu}-\partial_{\nu} A_{\mu} .
\end{aligned}
$$

It is a relativistic geodesic equation with respect to the affine connection (54).

Since the equation (70) coincides with the generic Lagrange equation (52) on a world manifold $X$, one can think of $H(69)$ as being a generic Hamiltonian of relativistic mechanics on $X$. 


\section{References}

[1] Echeverría Enríquez, A., Muñoz Lecanda, M. and Román Roy, N. (1991). Geometrical setting of time-dependent regular systems. Alternative models, Rev. Math. Phys. 3, 301.

[2] Giachetta, G., Mangiarotti, L. and Sardanashvily, G. (1997). New Lagrangian and Hamiltonian Methods in Field Theory (World Scientific, Singapore).

[3] Giachetta, G., Mangiarotti, L. and Sardanashvily, G. (2005). Geometric and Topological Algebraic Methods in Quantum Mechanics (World Scientific, Singapore).

[4] Giachetta, G., Mangiarotti, L. and Sardanashvily, G. (2006). Lagrangian and Hamiltonian dynamics of submanifolds, arXiv: math-ph/0604066.

[5] Giachetta, G., Mangiarotti, L. and Sardanashvily, G. (2009). Advanced Classical Field Theory (World Scientific, Singapore).

[6] Giachetta, G., Mangiarotti, L. and Sardanashvily, G. (2009). Advanced mechanics. Mathematical introduction, arXiv: 0911.0411.

[7] Krasil'shchik, I., Lychagin, V. and Vinogradov, A. (1985). Geometry of Jet Spaces and Nonlinear Partial Differential Equations (Gordon and Breach, Glasgow).

[8] De León, M. and Rodrigues, P. (1989). Methods of Differential Geometry in Analytical Mechanics (North-Holland, Amsterdam).

[9] Mangiarotti, L. and Sardanashvily, G. (1998). Gauge Mechanics (World Scientific, Singapore).

[10] Rovelli, C. (1991). Time in quantum gravity: A hypothesis, Phys. Rev. D43, 442.

[11] Sardanashvily, G. (1998). Hamiltonian time-dependent mechanics, J. Math. Phys. 39, 2714. 